\title{
IMPACTO DEL DISEÑO DE UNA PLATAFORMA VIRTUAL EN LA ENSEÑANZA DEL DERECHO
}

\section{IMPACT OF THE DESIGN OF A PLATFORM ONA VIRTUAL EDUCATIONAL COMMUNITY}

\author{
Franklin Steve Salazar Visurraga \\ Maestro en Ingeniería de Computación y Sistemas con mención en Gestión de TI \\ Universidad de San Martín de Porres \\ https://orcid.org/0000-0002-5701-0467 \\ fsalazarv@usmp.pe
} Perú

\section{SUMARIO}

- Introducción.

- El desarrollo de las TIC en la enseñanza.

- Los entornos virtuales como una oportunidad en la formación.

- La interactividad como factor clave en la enseñanza virtual del derecho.

- Importancia de una percepción colaborativa en la enseñanza virtual del derecho.

- Metodología de la investigación.

- Resultados.

- Discusión.

- Conclusiones.

- Recomendaciones.

- Fuentes de Información.

\section{RESUMEN}

La investigación se centra en determinar la influencia del nivel de calidad del diseño de una plataforma en el nivel colaborativo dentro de una comunidad educativa virtual en la enseñanza del derecho Se tomó como referencia la investigación "Diseño de una Comunidad Colaborativa en los Sistemas Educativos Virtuales Tradicionales", en la cual se midió el componente colaboración con dos tipos de sistemas educativos virtuales, teniendo como resultado que el nivel de colaboración era más alto en un tipo de sistema más flexible. La población seleccionada fue la Facultad de Derecho de la Universidad de San Martín de Porres de Perú, la cual brindó unos diplomados de carácter virtual- presencial para gente profesional. La presente investigación evaluó la posibilidad de aproximarse más a las razones de tener un ambiente colaborativo considerando otros elementos que pudieran influir en un trabajo asociativo dentro de una comunidad educativa virtual en la enseñanza del derecho. Por tal motivo se rescataron otras dimensiones para analizar, considerando la dimensión "diseño de plataforma" como prioridad, la cual fue medida desde la percepción de los participantes también y con ello ver la relación del nivel de calidad de la dimensión Diseño de Plataforma y la dimensión Comunidad Colaborativa definida en la investigación citada como referencia.

\begin{abstract}
The research focuses on determining the influence of the quality level of the design of a platform on the collaborative level within a virtual educational community in the teaching of law. The research "Design of a Collaborative Community in Traditional Virtual Educational Systems was taken as a reference", in which the collaboration component with two types of virtual educational systems was measured, with the result that the level of collaboration was higher in a more flexible type of system. The selected population was the Faculty of Law of the University of San Martín de Porres in Peru, which offered virtual-face-to-face diplomas for professional people. The present investigation
\end{abstract}


evaluated the possibility of getting closer to the reasons for having a collaborative environment considering other elements that could influence an associative work within a virtual educational community in the teaching of law. For this reason, other dimensions were analyzed to analyze, considering the dimension "platform design" as a priority, which was measured from the perception of the participants as well, and with it, see the relationship between the quality level of the Platform Design dimension and the Collaborative Community dimension defined in the research cited as a reference.

\section{PALABRAS CLAVES}

Comunidad, colaboración, plataforma, escenario virtual, alumno, docente, enseñanza, aprendizaje y derecho.

\section{KEYWORDS}

Community, collaboration, platform, virtual stage, student, teacher, teaching, learning and law.

\section{INTRODUCCIÓN}

El concepto de sociedad de la información está cobrando fuerza cada vez más en estos tiempos; y más aún, con las necesidades que el mundo vive por la pandemia que se ha suscitado. El ser humano necesita de la información y de su entorno para poder desarrollar conocimiento en las distintas áreas; y las TIC se consolidan como el camino emergente de reinvención en esta crisis. Desde tiempos remotos el ser humano necesitó de un entorno colaborativo para fortalecer sus propuestas $\mathrm{y}$, por ende, los medios para llegar a más personas fueron evolucionando progresivamente.

Las tecnologías de la información y la comunicación significan, hoy en día, un gran soporte para todos nosotros. Nos permite gestionar procesos que por la coyuntura actual hubiera sido difícil tratarlos de forma presencial. Muchos de ellos tratados de manera presencial contaban con un soporte virtual, el cual no fue puesto a prueba sino hasta hoy, un momento en el cual la sociedad está siendo afectada en diversos sectores. Con lo acontecido en la actualidad, algunas empresas tuvieron que adaptarse abruptamente a cambios en su organización, lo cual supuso una reingeniería en sus procesos más críticos para conservar un buen funcionamiento o alcanzar soluciones más efectivas ante la demanda constante de clientes o usuarios.

Los antecedentes de las TIC nos muestran una interacción evolutiva y constante con el ser humano, el cual de forma creciente hizo parte de su vida cotidiana el uso de soluciones tecnológicas para mejorar resultados. Como un ejemplo latente en estos tiempos tenemos a los dispositivos móviles, los cuales llegaron a convertirse en una alternativa más para tratar la información y comunicarnos de distintas formas.

Podríamos explicar el nacimiento de las TIC desde dos dimensiones. Una de ellas puede reflejarse desde la aparición de las primeras representaciones de sistemas computacionales en la década de los cincuenta del siglo pasado. Desde esa época se avizoró un camino en el cual las innovaciones tecnológicas iban a trascender en nuestras actividades de toda índole.

$\mathrm{Si}$ bien es cierto que las primeras computadoras estaban destinadas para ciertas ramas como la investigación o trabajos específicos con personas especializadas, siempre se contemplaba la posibilidad de una interacción superior con ellas. Los sistemas computacionales en sus distintas formas significaron toda una revolución en la gestión de información debido a que gradualmente fue introduciéndose en nuestras labores cotidianas un creciente interés por descubrir más de ellas; pero a su vez se percibía cierta resistencia por un sector reacio a involucrarse en nuevas formas de tratar la información, debido a diversos factores como la falta de conocimiento o el miedo a ser reemplazados.

Estas representaciones tecnológicas, que aparecieron como soluciones para agilizar el trabajo de las empresas y usuarios, fueron encajadas en el concepto de tecnologías de la información debido a que significaron una nueva manera de gestionarlas para tomar mejores decisiones en menor tiempo.

Las tecnologías de la información fueron potenciadas con la aparición de Internet. Esta tecnología se originó aproximadamente en inicios de los años sesenta del s. XX, debido a que fue contemplada como una estrategia militar para acceder a información confidencial. 
La inserción de las TIC en los procesos de nuestra sociedad fue una respuesta a las necesidades nuevas en distintos campos. El campo educativo fue uno de ellos, que necesitó poco a poco albergar una comunidad más interactiva y encontró en las TIC el puente ideal para unir sociedades mediante la aparición de las plataformas virtuales.

Son muchas las ramas de la educación que se han visto beneficiadas con el aporte de las TIC, específicamente con el surgimiento de plataformas que brindan un escenario donde el usuario puede encontrar información y compartirla. La información, como elemento primordial para tomar decisiones en corporaciones o empresas, no resulta ajena en las instituciones educativas desde el punto de vista de la construcción del conocimiento.

Parte de la construcción de conocimiento involucra la interacción, la cual actúa como factor clave para transmitir experiencias en distintos escenarios y de tal forma debería aplicarse en los entornos virtuales destinados a soportar la educación en distintas modalidades. Estas modalidades han sido insertadas en las instituciones educativas progresivamente en el tiempo pese a la desconfianza que envuelve a este sistema.

Los entornos virtuales orientados a soportar la educación en sus distintos niveles tienen el deber de prestar el mejor escenario enfocado en el estudiante, quien es el elemento en todo el sistema más importante. A pesar de tener claro esta idea y de que la crisis que estamos viviendo a raíz del Covid-19 demanda apostar por las TIC en la educación mediante el uso de las plataformas virtuales, los entornos virtuales enfrentan cierto rechazo para ser considerados como parte de los caminos de enseñanza.

Algunos factores para desestimar el uso de entornos virtuales en el campo educativo pueden estar relacionado a la falta de conocimiento de uso de recursos de la plataforma por parte de los docentes, a la poca participación de los alumnos debido a una percepción de nivel de colaboración muy bajo, etc.

Más allá de los inconvenientes que el uso de las plataformas virtuales en la educación puede presentar, la UNESCO (1998) rescató la innovación en la enseñanza y definió a los entornos virtuales de aprendizaje como un programa informático interactivo de carácter pedagógico que posee una capacidad de comunicación integrada, es decir, que su estructura esta en base a las tecnologías emergentes que estaban desarrollándose para potenciar la enseñanza. En ello se destaca la interacción como la manera más genuina de aprender y la base para construir conocimientos en entornos virtuales de aprendizaje.

\section{EL ROL DE LAS TIC EN LA ENSEÑANZA}

Actualmente, las TIC no solo significan una alternativa para mejorar los procesos de las empresas o agilizar diversas labores cotidianas, también implican una necesidad latente para poder sobrevivir. Las dificultades presentadas por la pandemia pueden suponer que el mundo o parte de él no estaba preparado para enfrentar un golpe tan fuerte; no obstante, las organizaciones tomaron un respiro para refundar sus ideas y reinventarse teniendo como respaldo a las TIC, las cuales nacieron para evolucionar y sostener los procesos que forman parte de nuestra sociedad independientemente del nivel de recursos que se tenga en distintas realidades.

Tras diversos intentos por salir del quiebre socioeconómico, se puede rescatar aspectos positivos los cuales están relacionados con el impulso a la digitalización. Si bien es cierto que antes de la pandemia estábamos inmersos en un mundo digital que prosperaba, hoy en día el uso de las TIC deja de ser una opción para convertirse en elemento vital para sostener la economía mundial. El aislamiento social obligatorio que se impuso como una medida de protección a las personas generó que se diera una mayor relevancia a tecnologías que se venían usando, así como también se dio espacio a otras nuevas por la necesidad de cambio.

El concepto de las TIC se compone de la evolución de las tecnologías de información y tecnologías de comunicación. Las tecnologías de la información fueron potenciadas con la aparición de Internet, lo cual propició que se fuera definiendo un camino distinto para establecer interacciones que fortalecieran los procesos de las organizaciones.

la información y la comunicación; y esto se debe a que no solo existían innovaciones que permitían de manera específica gestionar la 
información para tomar mejores decisiones, sino que también podían apreciarse herramientas que nos permitían transmitir la información hacia otros puntos mejorando la comunicación. Este nuevo modelo otorgó a las empresas el camino para extender sus negocios a lugares impensados anteriormente.

El campo educativo no fue ajeno a esta revolución, debido a que paulatinamente aprovechó las herramientas tecnológicas para consolidar sociedades más grandes de conocimiento. Poco a poco, los entornos educativos presenciales se sostenían en las TIC para presentar nuevas alternativas de trabajo en la enseñanza, proporcionando un nuevo espacio para acceder a la información. Con respecto a ello, Jesús Salinas Ibáñez (2004) afirma que:

Las perspectivas de las Tecnologías de la
Información y Comunicación (TIC) para la
formación, vienen motivadas tanto por
los avances de las telecomunicaciones y
las tecnologías de la información, como
por las transformaciones que en el campo
de la enseñanza se van dando por efecto
de integración y/o adaptación de dichas
tecnologías en el marco educativo. La
utilización de las TIC ofrece un amplio
abanico de posibilidades constituyendo un
reto para la enseñanza. (Pág. 1)

Al proponer un entorno virtual se debe tener en cuenta las distintas situaciones que pueden presentarse a los participantes y considerar el mejor camino para que la adaptación al uso de los recursos de la plataforma no genere vacíos en el logro de objetivos académicos.

En los últimos años los proyectos de las instituciones educativas fueron incorporando propuestas de nuevos escenarios cuyo soporte se basaba en tecnologías más fuertes, las cuales podrían denominarse plataformas virtuales.

Más allá de que las plataformas virtuales no siempre presentaron un escenario colaborativo para el proceso de enseñanza-aprendizaje, se puede decir que la evolución del ambiente que proponían fue generando adeptos a la modalidad que representan. La modalidad virtual fue cautivando a las personas por las ventajas que ofrecían de acceder a nuevos conocimientos desde cualquier punto. Si bien es cierto, las plataformas virtuales representan a las TIC en el campo educativo como una innovación en constante desarrollo, siempre hubo algún sector reacio a su incorporación en las estrategias educativas de cualquier rama.

Al originarse la pandemia del Covid-19 en nuestro país dejó muy sensible el área de educación en distintos sectores, lo cual ha provocado que las instituciones educativas reformulen su manera de abordar el proceso de enseñanza apoyándose en las TIC. Por tal motivo el uso de las plataformas virtuales se consolidó para poder continuar con los programas educativos. No obstante, varias universidades están presentando problemas técnicos al momento de respaldar un gran número de interacciones; a pesar de ello, la modalidad virtual apunta a ser considerada con mayor fuerza en los futuros programas educativos.

Los avances tecnológicos asociados al campo educativo tienen la misión de conectar a las personas y con ello formar una comunidad basada en la interacción constante. Las relaciones entre las personas siempre han consolidado nuevos modelos conceptuales; y con las tecnologías se fue extendiendo más las sociedades de conocimiento mediante escenarios virtuales.

\section{LOS ENTORNOS VIRTUALES COMO UNA OPORTUNIDAD EN LA FORMACIÓN}

Los entornos virtuales nacen como una alternativa en la enseñanza de modalidad presencial. Con el tiempo fueron consolidándose como el camino para llegar a más información de interés para desarrollar un nuevo conocimiento. Los entornos virtuales suponen un escenario en el cual se percibe una comunicación dinámica entre los participantes, pero el proceso para llegar a ese punto implica considerar más de un factor.

La evolución de los entornos virtuales significó una respuesta a la necesidad de acceder a nuevos modelos conceptuales no solo a nivel de contenido, sino también a nivel de interacción. Por ello se desarrollaron plataformas más robustas para soportar una mayor comunicación entre los usuarios involucrados, independientemente del tamaño de la comunidad que alberga. 
Con la consolidación de Internet en nuestra sociedad se pudo entender el concepto de tecnologías de Antes de esta crisis propiciada por el Covid-19, los entornos virtuales no fueron puestos a prueba en toda su magnitud por las instituciones educativas. Como se mencionó anteriormente, dichos escenarios eran considerados parcialmente, solo como un apoyo a la educación presencial. A raíz de la pandemia que se está viviendo en esta época, las instituciones educativas han tenido que apostar por las plataformas virtuales para mantener la continuidad de la enseñanza.

Dentro de todo el proyecto de resurgimiento en el campo educativo, son quizás las universidades $\mathrm{o}$ instituciones educativas de nivel superior quienes afrontan un mayor reto ante la ola de críticas debido al relativo soporte que brindan a toda una comunidad o por el hecho de la poca confianza de un sector con respecto a la modalidad virtual.

Más allá de la complejidad que implica introducir la modalidad virtual en los procesos de enseñanza, esta coyuntura debe significar una oportunidad para consolidar el salto a la digitalización. Las TIC nacieron para brindar soluciones y mejorar los procesos en nuestro entorno; y las plataformas virtuales son fieles representantes de estas soluciones. Tienen que ser bien gestionados para proyectar sostenibilidad en el futuro.

Los entornos virtuales educativos también implican una adaptación a cambios en la manera de transmitir conocimiento. Mason y Kaye (1990) señalaban que:

La aplicación de la comunicación mediada por ordenador estaba haciendo cambiar la naturaleza y estructura de las instituciones coetáneas de educación a distancia de diferentes formas, e indicaban tres implicaciones de dicho uso:

- La desaparición de las distinciones conceptuales entre la educación a distancia y la educación presencial.

- El cambio de los roles tradicionales del profesorado, tutores adjuntos y staff administrativo y de apoyo.

- Proporcionar una oportunidad, que nunca existió antes, de crear una red de estudiantes, un "espacio" para el pensamiento colectivo y acceso a los pares para la socialización y el intercambio ocasional. (Pág. 15 - 38)
Definitivamente existe un cambio con relación a los roles tradicionales en los entornos virtuales educativos. El docente tiene que tener el conocimiento básico para poder aprovechar los recursos de las plataformas virtuales y con ello ser un guía que propicie la interacción entre los estudiantes que participan en el escenario virtual.

El ser humano necesita de su entorno para formar nuevos modelos de conocimiento. En un escenario presencial el ser humano se relaciona con los demás participantes para esclarecer sus dudas y desarrollar nuevas ideas. Los entornos virtuales no pueden ser ajeno el hecho de mantener la conectividad, pero involucra la consideración de una serie de elementos que invitan a ser investigados de forma independiente como el rol del docente, su nivel de conocimiento, la flexibilidad de la plataforma virtual, etc.

Collis y Moneen (2001) por su parte rescatan la flexibilidad del aprendizaje en la educación superior considerando cuatro componentes:

- Tecnología que se refiere tanto a los ordenadores y redes, como a las herramientas y aplicaciones de software. Es decir, "aplicaciones tecnológicas" cuyo uso educativo puede ser publicación y diseminación de la información, comunicación, colaboración, tratamiento de la información y los recursos, así como propósitos específicos de enseñanza y aprendizaje o integración de cursos.

- Pedagogía, que se ocupa del enfoque o de los modelos pedagógicos, la orientación de las actividades, marco de flexibilidad de estas, etc.

- Estrategia de implementación relacionada con los factores que caracterizan la innovación.

- Marco institucional. (Pág. 9 - 10)

La búsqueda de calidad en los entornos virtuales ha sido una constante y los enfoques para priorizar el elemento determinante a potenciar para darle un mejor escenario al estudiante dependieron de las políticas de cada institución educativa. Lo que no se puede perder de vista es que en todo el proceso de enseñanza el estudiante debe ser el más beneficiado. 
La crisis que se está viviendo en nuestro país de alguna manera nos da la oportunidad de aprovechar las TIC en la educación de cualquier rama mediante el uso de las plataformas virtuales y con ello potenciar el salto a una era en la cual se demanda una conectividad constante con la información y capacitación progresiva en el uso de nuevas herramientas tecnológicas para desarrollar nuevos caminos de búsqueda de conocimiento.

\section{LA INTERACTIVIDAD COMO FACTOR CLAVE EN LA ENSEÑANZA VIRTUAL DEL DERECHO}

Debido a la coyuntura que estamos atravesando, las instituciones educativas de nivel superior han tenido que recurrir al uso de las tecnologías para mantener su labor de enseñanza. Ello ha generado que previamente se reestructuren los planes y se desarrollen capacitaciones para fortalecer el conocimiento sobre el uso de los recursos que puede tener una plataforma virtual para soportar las interacciones durante un periodo académico.

Las interacciones que pueden desarrollarse en un ámbito laboral son importantes para la solución de problemas, de igual forma para alcanzar niveles más altos de conocimientos. Esto se refleja en el campo educativo también, en el cual los estudiantes mediante la participación, intercambio de ideas o dinámicas de clase llegan a comprender mejor un tema de discusión. Los escenarios virtuales deben conservar el mismo sentido de comunidad, en el cual el estudiante puede tener a su entorno como aliado para poder aprender constructivamente.

En un principio los entornos virtuales eran concebidos como repositorios, que, si bien permitía al estudiante acceder a información, no albergaba la posibilidad de soportar ninguna comunicación entre los participantes. Esto fue mejorando con los años porque se desarrollaron entornos más amigables y flexibles para establecer dinámicas de trabajo colaborativo.

La pandemia que el mundo está viviendo nos está recordando el cambio de los escenarios en la educación, debido a que las instituciones educativas han tenido que apresurar su adaptación a entornos digitales que en un principio parecían muy lejos de ser un elemento vital para sostener el proceso de enseñanzaaprendizaje como lo señala Coll. (2012).

El nivel de flexibilidad en los escenarios virtuales es importante en todas las ramas educativas porque permite mantener una conexión con la información actualizada, la cual puede venir de contenidos digitales o de las mismas relaciones con otros participantes en las diversas actividades que pueden establecerse.

El presente artículo rescata la enseñanza del derecho como un claro ejemplo donde las interacciones son esenciales para la formación del estudiante. Es una rama donde se discute constantemente las ideas para desarrollar las competencias del futuro profesional

Tal como lo recalcan Muirhead y Juwah, (citados por Peñalosa y Castañeda, 2010 p.1182) "En los entornos virtuales de aprendizaje, la interactividad es un aspecto fundamental, para potenciar los procesos de aprendizaje autónomo, tanto en la apertura del diálogo académico entre los estudiantes, como en el acompañamiento que hace el tutor al proceso formativo de sus alumnos".

Esto es importante cuando se desarrolle la enseñanza del derecho en un escenario virtual y debe verse potenciado por el conocimiento de recursos por parte del docente para guiar a los estudiantes para no perder la esencia que caracteriza a esta rama. Adicional a ello, debe conservarse la interactividad en las actividades programadas o propuestas.

Es esencial mantener la interactividad para fortalecer el sentido de comunidad colaborativa en la formación y conservar el nivel participativo que suele percibirse en un entorno presencial. Se puede sustentar la importancia de fomentar la interactividad en los siguientes puntos:

- Dos Santos (2012) considera que la utilización de los medios visuales en un entorno educativo virtual permite al estudiante organizar mejor su aprendizaje, profundizando la idea central del tema abordado. La influencia de la flexibilidad de la plataforma y de los medios audiovisuales con los cuales interactúa el estudiante favorece al aprendizaje que pueda desarrollar. 
- La experiencia y las relaciones que se tienen con el entorno fortalecen el aprendizaje de los estudiantes, como lo señaló Collins y Halverson (2009).

- Los contenidos interactivos le dan calidad a la modalidad virtual en la educación como lo sostuvo Karahoca (2010).

- Según Albuquerque (2010), la interactividad de los estudiantes en un escenario virtual proporciona un sentido de acompañamiento al estudiante para poder interiorizar mejor los temas que se establecen en un programa educativo virtual.

Esta nueva era digital que está consolidándose poco a poco en tiempos de crisis, invita a las instituciones educativas de nivel superior a adaptarse a los cambios y considerar recursos que quizás en un momento no contemplaban explotar por antiguos paradigmas que la misma sociedad impuso por un sector. Investigaciones relacionadas a la enseñanza del derecho advertían nuevas necesidades de cambio para continuar con la formación de estudiantes.

Juan José González Rus (2003) sostiene con relación a la enseñanza del derecho mediante el uso de las TIC que:

Al margen de cuál sea el futuro de la enseñanza del derecho y de que se haga o no algo al respecto, lo que parece inaplazable es asumir que el futuro va a plantear nuevas necesidades a la enseñanza del derecho que no ser ignoradas. Se considera muchas, pero de entre todas ellas solo hay dos que parecen más urgentes y principales:

- Incorporación progresiva de las nuevas tecnologías, Internet y las redes de transmisión de datos a la gestión y organización de la docencia y al propio desarrollo de las actividades docentes, tanto propias de la licenciatura como posteriores a la misma.

- Diseño estratégico de las actividades docentes por las Facultades de Derecho de forma diversificada, orientadas a dos cometidos básicos: los estudios correspondientes a las licenciaturas que impartan y la oferta formativa posterior a las mismas.

Parte del cambio que se estimaba hace un tiempo en la enseñanza del derecho tiene que ver no solo con la incorporación de nuevas tecnologías, sino con las acciones respectivas para promover la interactividad en los entornos virtuales para poder desarrollar las competencias de los estudiantes.

La interactividad es inherente al concepto de trabajo colaborativo y debe ser considerado como elemento base en un entorno virtual.

Lukman y Krajnc (2012), consideraron que el trabajo colaborativo permitía a los estudiantes generar nuevas ideas, producto del debate $\mathrm{y}$ apoyo constante de los participantes, quienes mediante las actividades en los entornos virtuales reforzaban sus dudas y desarrollaban nuevos conocimientos.

Por lo tanto, puede definirse el trabajo colaborativo como una estrategia conveniente para la enseñanza del derecho en los entornos virtuales, debido a que permite optimizar la gestión del tiempo por el apoyo de los demás participantes o estudiantes pertenecientes al programa educativo virtual, adquiriendo habilidades cognitivas y metacognitivas, tal cual lo afirmaba Mariac Moreno-Salamanca (2013).

\section{IMPORTANCIA DE UNA PERCEPCIÓN COLABORATIVA EN LA ENSEÑANZA VIRTUAL DEL DERECHO}

Los entornos virtuales muestran una serie de variantes de acuerdo al diseño que tienen las plataformas que actúan como soporte de toda la interacción. A lo largo del tiempo, la evolución de los entornos virtuales fue marcándose por el nivel comunicación que sus herramientas permitían generar.

En un principio se podían apreciar como repositorios donde se guardaba el contenido para el acceso de los participantes de una materia, pero posteriormente fueron reforzándose las tecnologías de información y comunicación para permitir una interacción más sólida e insertar la sensación de acompañamiento.

Como se mencionó anteriormente, mantener la interactividad es una misión clave en los entornos educativos virtuales para consolidar el sentido de colaboración entre los estudiantes teniendo al docente como guía. La presente investigación considera el diseño de una plataforma virtual como un elemento importante que acondiciona el trabajo colaborativo en la enseñanza virtual del derecho. 
A pesar de que los modelos de enseñanza en un inicio se caracterizaban por solo la exposición del docente, poco a poco fue incorporándose medios para incentivar la participación de los estudiantes y poder con ello desarrollar competencias para poder enfrentar el mundo laboral. En base a ello, la evolución de los entornos virtuales fue señalando la importancia de mantener conectados a los alumnos mediante los recursos que integraban las plataformas.

Como lo anticipaban Harasim, Hiltz, Teles y Turoff (1995), las redes de aprendizaje son el modelo de educación del siglo XXI, en el cual los estudiantes cooperan entre si dentro de un escenario virtual para desarrollar nuevos conocimientos a pesar de estar en lugares distintos. A esto se suma lo enunciado por Garrison y Anderson (2005), quienes consideran la interactividad como la base de la red de aprendizaje, la cual en el mundo contemporáneo se puede conocer como comunidad virtual de aprendizaje basada en la colaboración.

\section{Merinhos \& Osório (2009) sostienen que:}

El aprendizaje colaborativo en ambientes virtuales establece una nueva relación entre los participantes del proceso de aprendizaje, una vez que el aprendizaje colaborativo es más exigente que el aprendizaje individual. En el ambiente virtual, la colaboración, requiere una mediación tecnológica y el alargamiento en el espacio y en el tiempo del relacionamiento y, por otro lado, requiere un proceso interactivo intenso y regular de envolvimiento cognitivo (Pág. 45 - 60).

La concepción de lo que hoy significa las comunidades virtuales de aprendizaje como el camino para construir conocimiento tiene su base en las teorías socioeducativas basadas en el aprendizaje social como lo señalaban Edith Inés Ruíz Aguirre, Nadia Livier Martínez de la Cruz y Rosa María Galindo González (2015).

Edith Inés Ruíz Aguirre, Nadia Livier Martínez de la Cruz y Rosa María Galindo González (2015) resumen ciertos elementos que una plataforma que soporta una comunidad virtual de aprendizaje debe permitir desarrollar:

a) Accesibilidad: se entiende como la posibilidad de que los integrantes puedan establecer los canales de comunicación correspondientes para que se lleve a cabo el flujo de la información a través de la computadora. b) Participación: es un elemento necesario para el flujo de la información ya que involucra la colaboración e intercambio de ideas de los integrantes, aceptación de las ideas, la diversidad social y la disposición de compartir el conocimiento o las experiencias, así como asumir el compromiso y responsabilidad social dentro del grupo.

c) Habilidades sociales y de gestión: estas habilidades abren la posibilidad de que los miembros de la comunidad puedan gestionar, aportar y generar el intercambio de ideas, mismas que contribuyen al procesamiento de la información $\mathrm{y}$, por ende, a la construcción del conocimiento.

d) Un contenido de discusión o construcción: dichos contenidos integran las reglas e información de calidad, concretas y claras a fin de conducir a conclusiones planeadas, intencionadas y generadoras de conocimiento (Pág. 83).

A raíz de conocer los elementos básicos que una plataforma debe permitir desarrollar para un aprendizaje colaborativo, el presente artículo tiene como objetivo medir el impacto del nivel de calidad del diseño de una plataforma virtual en el nivel de colaboración percibido por los estudiantes en la enseñanza del derecho.

\section{METODOLOGÍA DE LA INVESTIGACIÓN}

La investigación realizó un análisis cuantitativo para medir la media de las puntuaciones y la media con el fin de ver dispersión en los resultados.

También realizó un análisis cualitativo para describir las puntuaciones obtenidas. Estas puntuaciones fueron categorizadas en satisfecho e insatisfecho, con lo cual se utilizó la proporción como medida para interpretar los resultados.

\section{Método de la investigación}

Para el nivel de comunidad colaborativa se tomó en cuenta los resultados obtenidos de la encuesta realizada para la tesis "Diseño de una Comunidad Colaborativa en los Sistemas Educativos Virtuales Tradicionales". Esta tesis fue realizada por el propio autor y específicamente para la presente investigación 
se tomó la muestra relacionada al grupo de estudiantes de un diplomado de derecho.

Por lo tanto, para investigar el impacto del diseño de una plataforma virtual en la enseñanza del derecho teniendo como referencia a la tesis mencionada se desprendió dos aspectos muy importantes para el desarrollo del trabajo:

- Para medir el nivel de comunidad colaborativa se consideró el desarrollo planteado en la tesis mencionada.

- Para medir el nivel de calidad de diseño de plataforma, se realizó un análisis factorial para determinar que ítems de la encuesta definen la dimensión diseño.

\section{Diseño de la investigación}

El diseño de investigación fue transversal porque se realizó en un momento del tiempo, teniendo como instrumentos de recolección de datos el cuestionario y como técnica a la encuesta.

\section{Tipo de investigación}

El tipo de investigación es de naturaleza descriptiva debido a que se selecciona y describe una serie de aspectos relacionados con la influencia de la dimensión Diseño de Plataforma en la dimensión Comunidad Colaborativa en un tipo de sistema virtual considerado por la tesis de referencia como sistema tradicional.

Los resultados nos servirán para validar la relación de las dimensiones Comunidad Colaborativa y Diseño de Plataforma. Esta relación se menciona en la hipótesis más adelante con los resultados.

\section{Población y muestra}

Como se toma de referencia la tesis mencionada anteriormente para la presente investigación, se tiene en cuenta para el análisis la población relacionada al grupo de estudiantes que estudian un diplomado en la rama de derecho.
Tabla 1: Cuadro comparativo de perfiles de la población tomada en cuenta para el analisis.

\section{PERFIL DE LA POBLACIÓN}

Abogados, docentes universitarios, fiscales, jueces, universitarios, profesionales, estudiantes de postgrado y público en general.

EDAD PROMEDIO: 34 años

Fuente: Tesis "Diseño de una Comunidad Colaborativa en los Sistemas Educativos Virtuales Tradicionales", disponible en el repositorio USMP (http://repositorio.usmp.edu.pe/ handle/usmp/2747)

Elaboración; Propia

Las características del perfil de la población hacen referencia al siguiente grupo bajo un tipo de diseño de plataforma:

- Facultad de Derecho de la Universidad de San Martín de Porres

- Diplomados Virtual - Presencial 2014 (Agosto - Noviembre)

- Plataforma Moodle para soportar el aprendizaje virtual

Después de identificar la población, se determina la muestra en base a los resultados obtenidos de la encuesta relacionada a la tesis de referencia. Se determina 58 participantes como tamaño de muestra para realizar el análisis respectivo del impacto del nivel de calidad del diseño de plataforma sobre el nivel de comunidad colaborativa percibido por los estudiantes de derecho del diplomado mencionado.

\section{Técnicas de recolección de datos}

Con respecto a las técnicas de recolección de datos utilizadas se puede mencionar las que se utilizaron en el trabajo de la tesis mencionada, puesto que en ese periodo de tiempo se hizo la toma de datos para posteriormente trabajarlas en función de los objetivos trazados. Actualmente estos datos recogidos nos sirven teniendo en cuenta que la presente investigación pretende acercarse más a las razones que generaron un tipo de percepción con respecto al trabajo colaborativo en las muestras determinadas en aquel año. 
La investigación pretende establecer si existe relación entre el nivel de calidad del diseño de una plataforma virtual y el nivel de trabajo colaborativo en una comunidad educativa virtual. Para ello se utilizó las percepciones de los estudiantes.

\section{Descripción de los instrumentos}

Con relación a los instrumentos seleccionados, tenemos que mencionar los descritos en la investigación citada como referencia:

- Reportes digitales, proporcionados por la Unidad Virtual de la Universidad de San Martín de Porres.

- Plataforma Surveymonkey, la cual dio la posibilidad de elaborar las encuestas web respectivas para dirigirlas a los alumnos, ya sea vía correo electrónico o por medio de algún administrador de medio social.

- Encuestas físicas, que para algunas circunstancias se solicitó de esta forma, de acuerdo a los permisos y restricciones.

- Los medios sociales, en algunos casos se contactó con el administrador de Facebook de una de las universidades en estudio, para poder difundir las encuestas web.

\section{Técnicas para el procesamiento de la información}

Para el procesamiento se utilizará el software estadístico SPSS v23, con el que se obtendrá un análisis exploratorio de los datos (Frecuencias estadísticas y gráficos), para luego realizar el análisis con la variable (preguntas de interés Dimensiones de Colaboración y Diseño).

Para un mejor análisis se van a recodificar las puntuaciones de las variables para luego llevarlas a la dimensión, con la puntuación final ponderada entre las variables se clasificará en dos categorías (Satisfecho o Insatisfecho), para luego evaluarlo en cada grupo.

\section{RESULTADOS}

\section{Caracteristicas de la muestra}

Como se mencionó anteriormente, la investigación se basa en la información de la tesis "Diseño de una Comunidad Colaborativa en los Sistemas Educativos Virtuales Tradicionales", se toma una de las muestras para realizar el análisis respectivo. La muestra seleccionada corresponde al perfil de estudiantes de la rama de derecho, quienes llevaron un diplomado eligiendo una especialidad.

Los resultados muestran que $33(56.9 \%)$ de los encuestados son mujeres, y el $25(43.1 \%)$ son varones en el sistema educativo virtual considerado para el análisis de la presente investigación.

El perfil por rango etario en el escenario virtual que se considera como referencia concentra entre las edades de 24 -30 años con 26 (44.9\%) alumnos y de $31-37$ con 14 (24.1\%) alumnos,

Tabla $N^{\circ} 2$ : Características generales de la muestra seleccionada

\begin{tabular}{lc}
\hline \multicolumn{1}{c}{ MUESTRA } & ESCENARIO VIRTUAL \\
\hline Femenino & $33(56.9 \%)$ \\
Masculino & $25(43.1 \%)$ \\
\hline $17-23$ & $4(6.9)$ \\
$24-30$ & $26(44.8)$ \\
$31-37$ & $14(24.1)$ \\
$38-44$ & $8(13.8)$ \\
$45-$ más & $6(10.3)$ \\
\hline Muy poco & $3(5.2)$ \\
Poco & $26(44.8)$ \\
Medianamente & $20(34.5)$ \\
Mucho & $7(12.1)$ \\
Bastante & $2(3.4)$ \\
\hline
\end{tabular}

Fuente: Tesis "Diseño de una Comunidad Colaborativa en los Sistemas Educativos Virtuales Tradicionales", disponible en el repositorio USMP (http://repositorio.usmp.edu.pe/ handle/usmp/2747)

Elaboración: Propia

Se desprende del escenario virtual tomado para el análisis que solo $9(15.5 \%)$ de los encuestados ingresan mucho o bastante. 
Figura $\mathrm{N}^{\circ} 1$ : Detalle de las características generales de la muestra tomada.

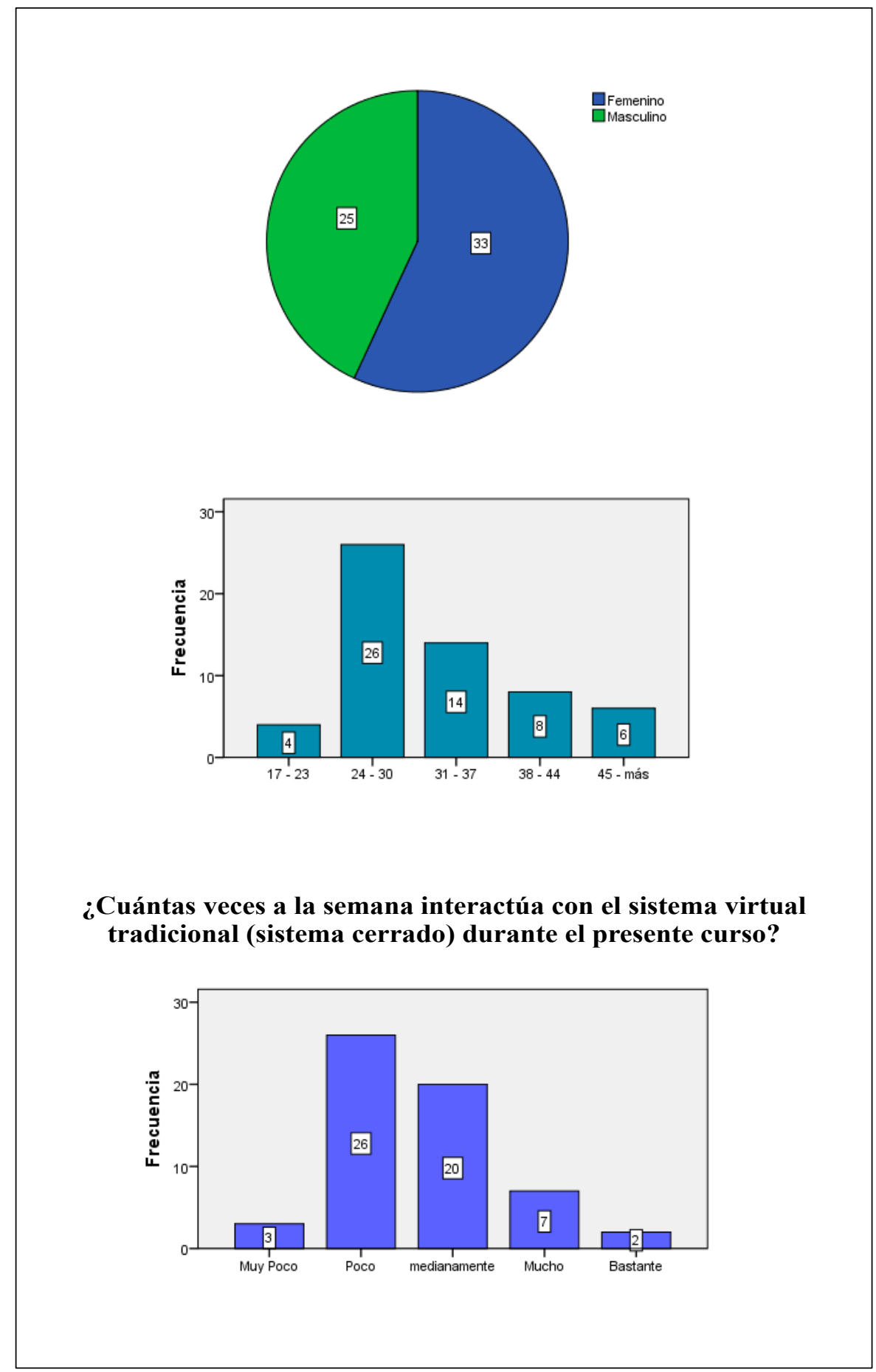

Fuente: Elaborado por autor. 


\section{Análisis factorial del diseño de la plataforma}

Para determinar la dimensión de Diseño de Plataforma, aplicamos el análisis factorial con la finalidad de probar que las 5 variables seleccionadas formen un mismo constructo que nombraremos "Diseño de Plataforma".

Como resultado del análisis factorial mostramos la matriz de varianza explicada, que muestra que estas 5 variables se están asociando a un solo constructo y que este explica el $73 \%$ de la variabilidad de esta variable subyacente "Diseño de la plataforma".

La matriz de componentes muestra los coeficientes (aporte de las variables al constructo). Las variables 1 y 3 presentan mayores índices porque aportan más en la variabilidad de la dimensión "Diseño de la Plataforma".

\section{Tabla $\mathbf{N}^{\circ}$ 3: Varianza total explicada}

Varianza total explicada

\begin{tabular}{|l|r|r|r|r|r|r|}
\hline \multirow{2}{*}{ Componente } & \multicolumn{4}{|c|}{ Autovalores iniciales } & \multicolumn{3}{c|}{ Sumas de las saturaciones al cuadrado de la } \\
extracción
\end{tabular}

Método de extracción: Análisis de Componentes principales.

Fuente: Desarrollado con el software SPSS por autor.

Figura N²: Gráfico de sedimentación

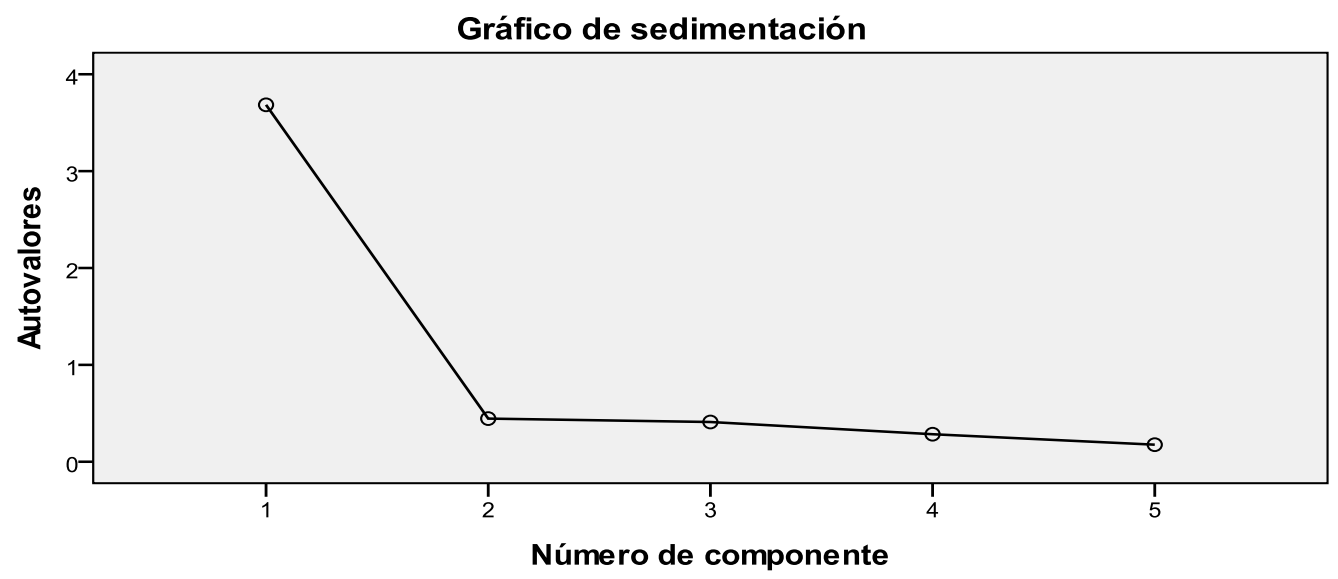

Fuente: Desarrollado con el software SPSS por autor. 
Tabla $N^{\circ}$ 4: Matriz de componente.

\begin{tabular}{|c|c|}
\hline & Componente \\
\hline & 1 \\
\hline $\begin{array}{l}\text { ¿Considera que la plataforma del curso que lleva le permite responder sin ninguna dificultad las } \\
\text { tareas asignadas? }\end{array}$ & ,864 \\
\hline ¿Considera que la plataforma le permite acceder sin problemas a descargas de contenidos? & ,804 \\
\hline ¿Considera que puede acceder sin problemas a la plataforma del curso? & ,916 \\
\hline ¿Considera que pudo acceder desde cualquier localidad a la plataforma del curso? & ,861 \\
\hline $\begin{array}{l}\text { ¿Considera que la plataforma del curso le permite aportar o generar temas de discusión para el } \\
\text { grupo de participantes? }\end{array}$ & ,844 \\
\hline
\end{tabular}

Método de extracción: Análisis de componentes principales.

a. 1 componentes extraídos

Fuente: Desarrollado con el software SPSS por autor.

\section{Análisis exploratorio}

Se realizará un análisis descriptivo de los ítems que componen cada una de las dimensiones (Comunidad Colaborativa y Diseño de
Plataforma) en el sistema educativo virtual que soporta la muestra tomada para el presente artículo

\section{Dimensión Comunidad Colaborativa}

Tabla $N^{\circ}$ 5: Estadísticas descriptiva de la valoración percibida en dimension - Comunidad Colaborativa

\begin{tabular}{|c|c|c|c|c|}
\hline Dimensión Colaboración & Media & Mediana & Mínimo & Máximo \\
\hline $\begin{array}{l}\text { ¿Considera se establecen foros de discusión en el sistema virtual } \\
\text { (plataforma) durante el curso para su debate? }\end{array}$ & 3.22 & 3.00 & 1.00 & 5.00 \\
\hline $\begin{array}{l}\text { ¿Considera se establece una comunicación fluida entre el } \\
\text { docente/moderador y alumnos mediante el sistema virtual } \\
\text { (plataforma)? }\end{array}$ & 3.17 & 3.00 & 1.00 & 5.00 \\
\hline $\begin{array}{l}\text { ¿Considera que sus compañeros en el sistema virtual } \\
\text { (plataforma) colaboran con usted para absolver sus dudas y } \\
\text { consolidar sus conocimientos? }\end{array}$ & 2.90 & 3.00 & 1.00 & 4.00 \\
\hline
\end{tabular}

Fuente: Desarrollado con el software SPSS por mi persona.

Se observa que la puntuación media en cada pregunta no dista mucho de su mediana con lo que la percepción es casi homogénea, salvo las dos primeras preguntas (establecen foros y comunicación fluida), cuyos valores mínimos y máximos son extremos. 
Figura $N^{\circ}$ 3: Comportamiento de las componentes de la dimensión - Comunidad Colaborativa

¿Considera se establecen foros de discusión en el sistema virtual (plataforma) durante el curso para su debate?

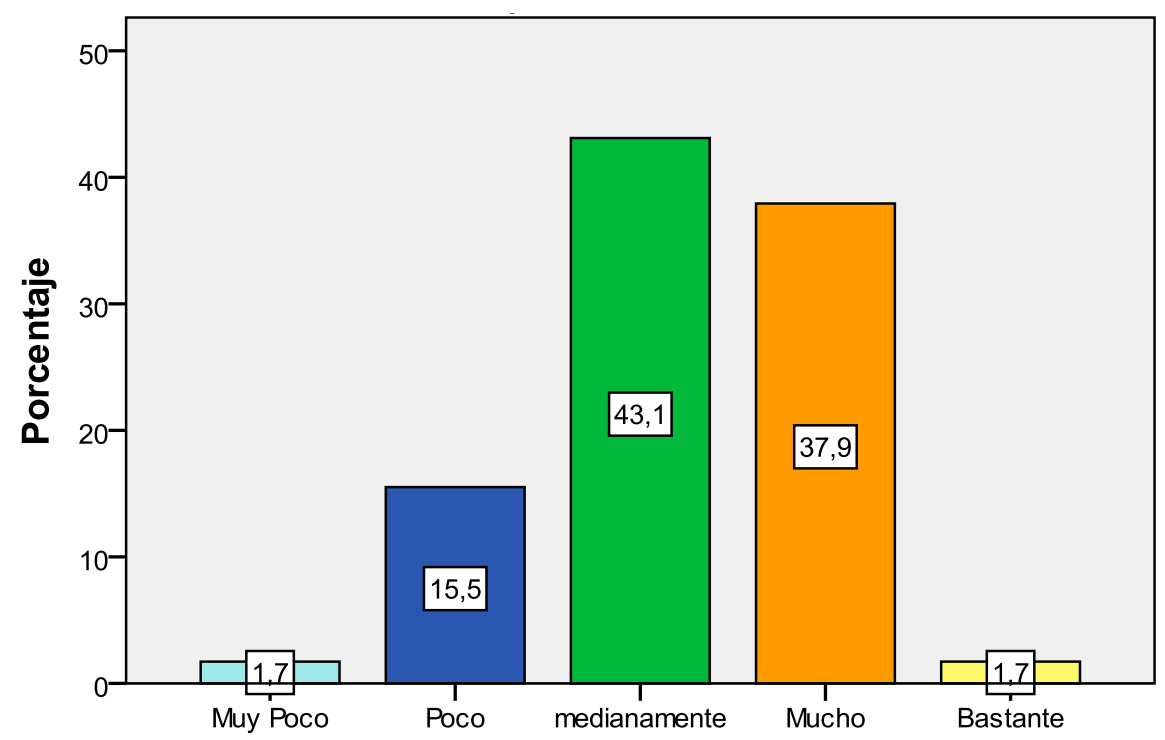

Fuente: Desarrollado con el software SPSS por mi persona.

¿Considera se establece una comunicación fluida entre el docente/moderador y alumnos mediante el sistema virtual (plataforma)?

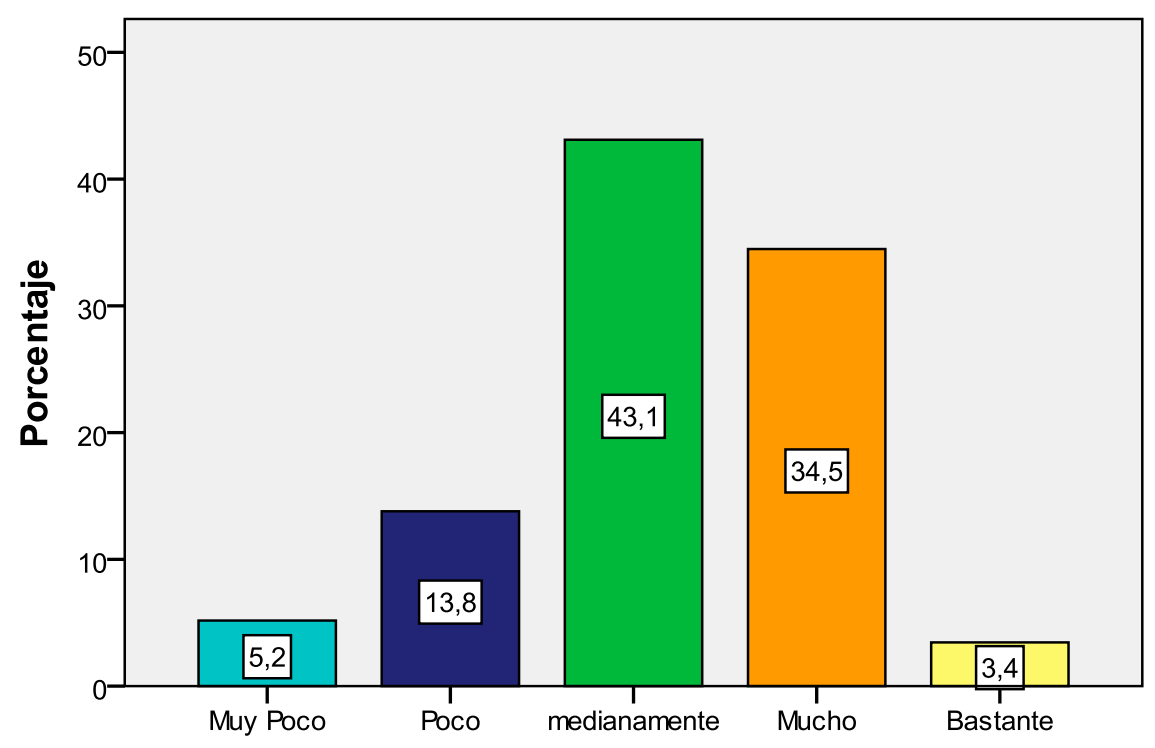




\section{¿Considera que los compañeros en el sistema virtual (plataforma) colaboran con usted para absolver sus dudas y consolidar sus conocimientos?}

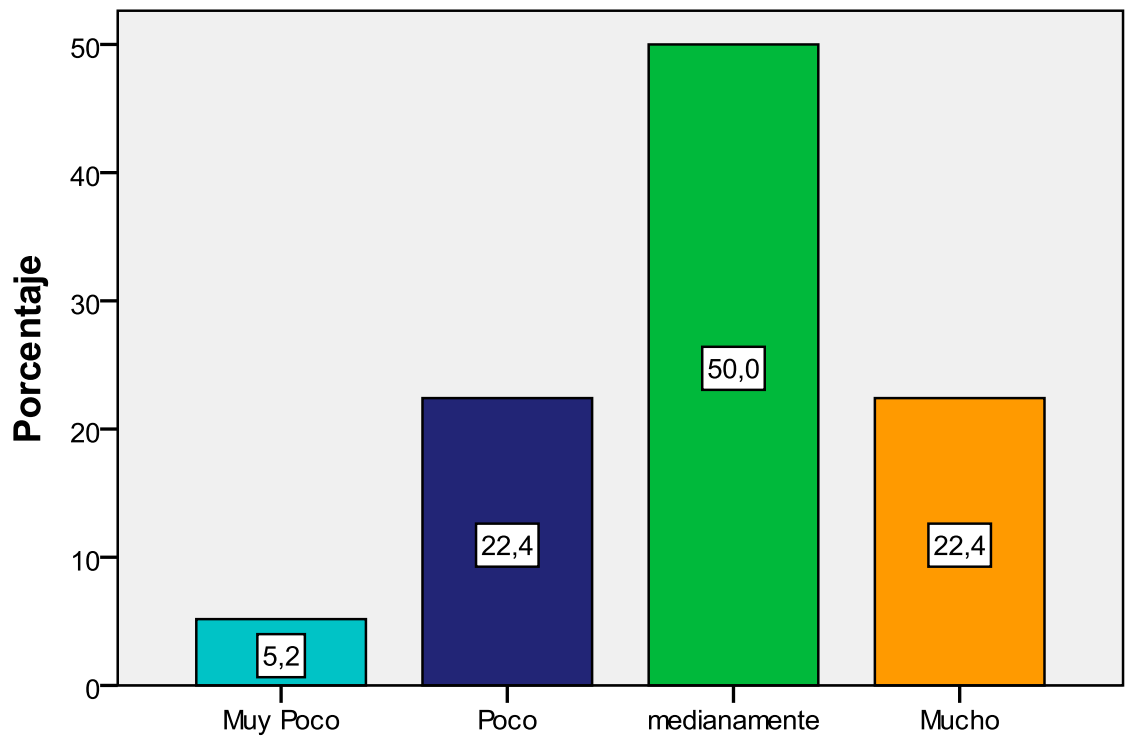

Fuente: Desarrollado con el software SPSS por autor.

Se aprecia que las puntuaciones se concentran en las categorías de (poco, medianamente y mucho), siendo la de mayor frecuencia medianamente, lo que en este sistema es más rígido y no se tiene muy desarrollado la dimensión de colaboración.

\section{Tabla $N^{\circ} 6$ : Estadísticas descriptivas de la valoración percibida de la dimensión - Diseño de Plataforma}

\section{Dimensión Diseño de Plataforma}

\begin{tabular}{|c|c|c|c|c|}
\hline Dimensión Diseño de Plataforma & Media & Mediana & Mínimo & Máximo \\
\hline $\begin{array}{l}\text { ¿Considera que el sistema virtual (plataforma) del curso que lleva } \\
\text { le permite responder sin ninguna dificultad las tareas asignadas? }\end{array}$ & 3.33 & 4.00 & 1.00 & 5.00 \\
\hline $\begin{array}{l}\text { ¿Considera que el sistema virtual (plataforma) le permite acceder } \\
\text { sin problemas a descargas de contenidos? }\end{array}$ & 3.61 & 4.00 & 1.00 & 5.00 \\
\hline $\begin{array}{l}\text { ¿Considera que puede acceder sin problemas al sistema virtual } \\
\text { (plataforma) del curso? }\end{array}$ & 3.55 & 4.00 & 1.00 & 5.00 \\
\hline $\begin{array}{l}\text { ¿Considera que pudo acceder desde cualquier localidad al sistema } \\
\text { virtual (plataforma) del curso? }\end{array}$ & 3.40 & 3.50 & 1.00 & 5.00 \\
\hline $\begin{array}{l}\text { ¿Considera que el sistema virtual (plataforma) del curso le } \\
\text { permite aportar o generar temas de discusión para el grupo de } \\
\text { participantes? }\end{array}$ & 3.36 & 3.00 & 1.00 & 5.00 \\
\hline
\end{tabular}

Fuente: Desarrollado con el software SPSS por mi persona. 
Se observa que la puntuación media y mediana en cada pregunta no dista mucho, con lo que la percepción es casi homogénea, sin embargo, la pregunta dos y tres (Descarga y Acceso), presenta menor diferencia entre sus medias y medianas.

\section{Figura $N^{\circ}$ 4: Comportamiento de los componentes de la dimensión - Diseño de Plataforma}

¿Considera que la plataforma del sistema virtual (plataforma) le permite acceder sin problemas a descargas de contenidos?

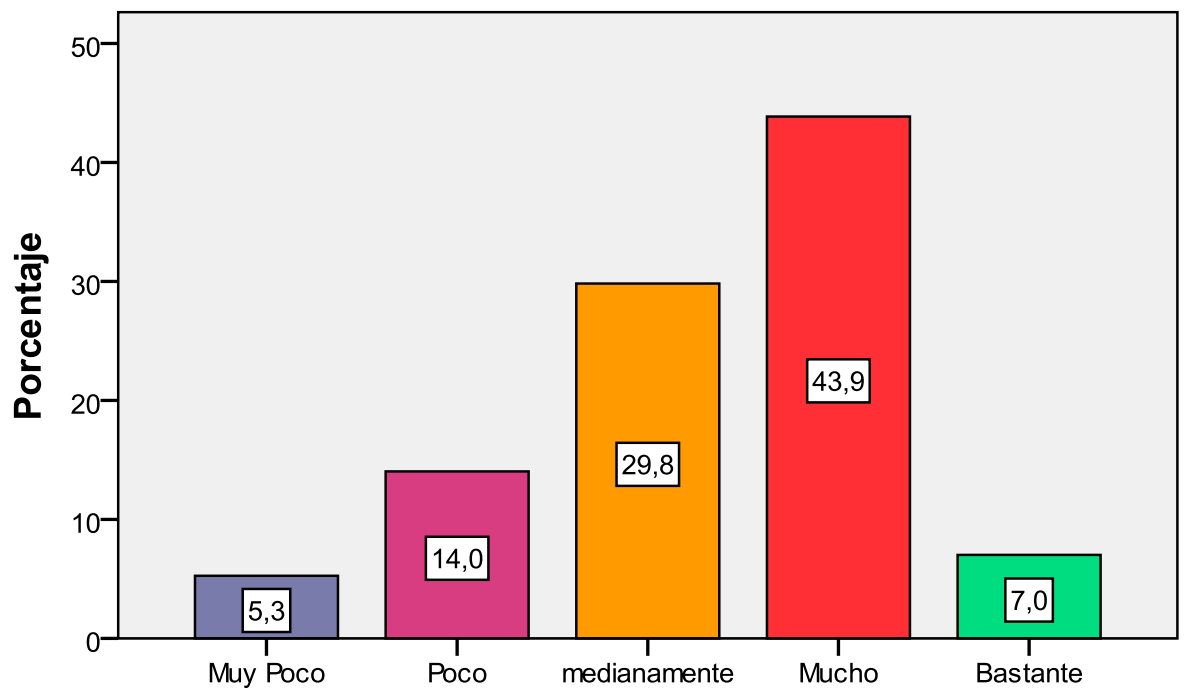

¿Considera que pudo acceder desde cualquier localidad al sistema virtual (plataforma) del curso?

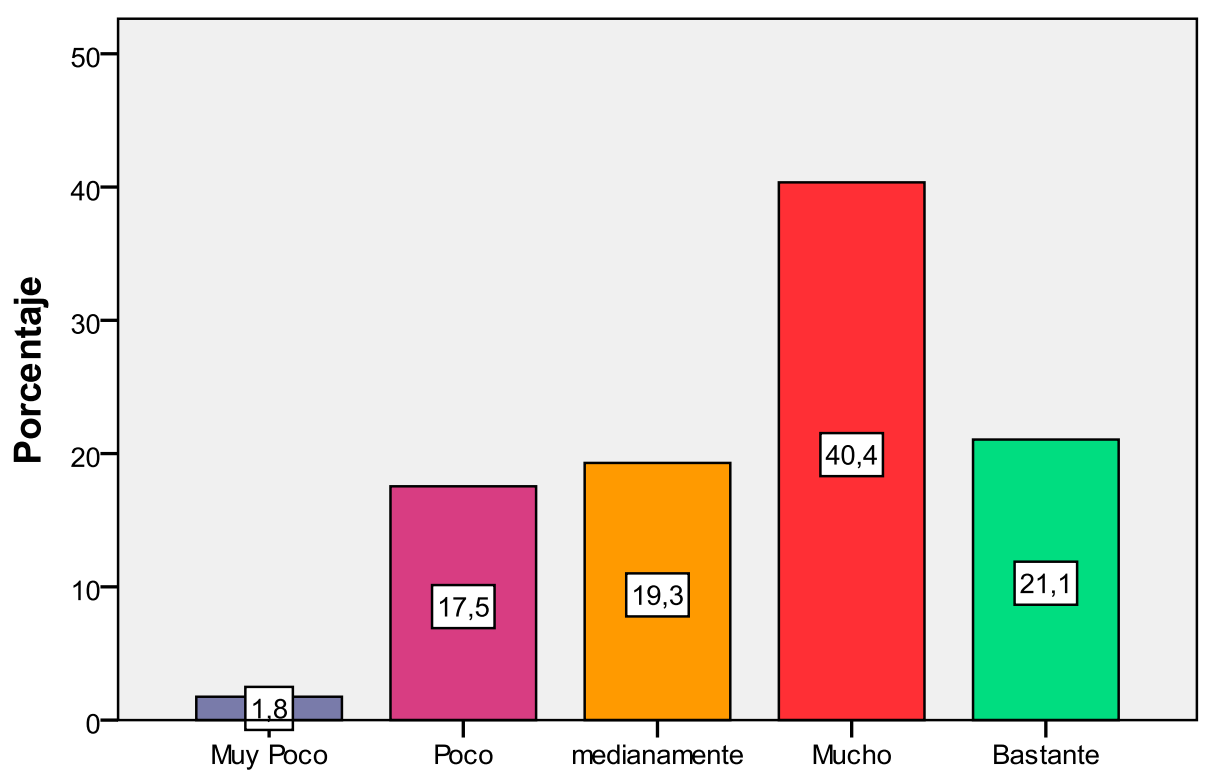




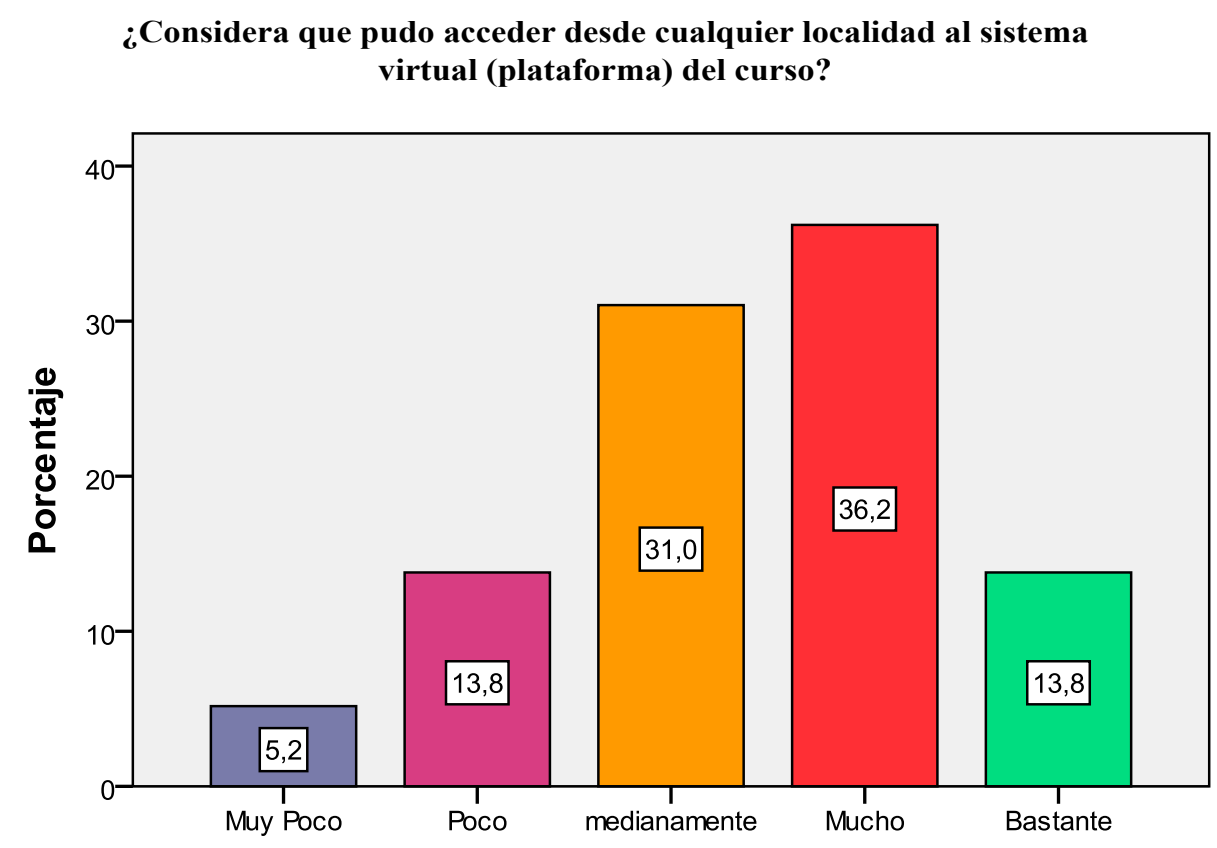

¿Considera que el sistema virtual (plataforma) le permite aportar o generar temas de discusión para el grupo de participantes?

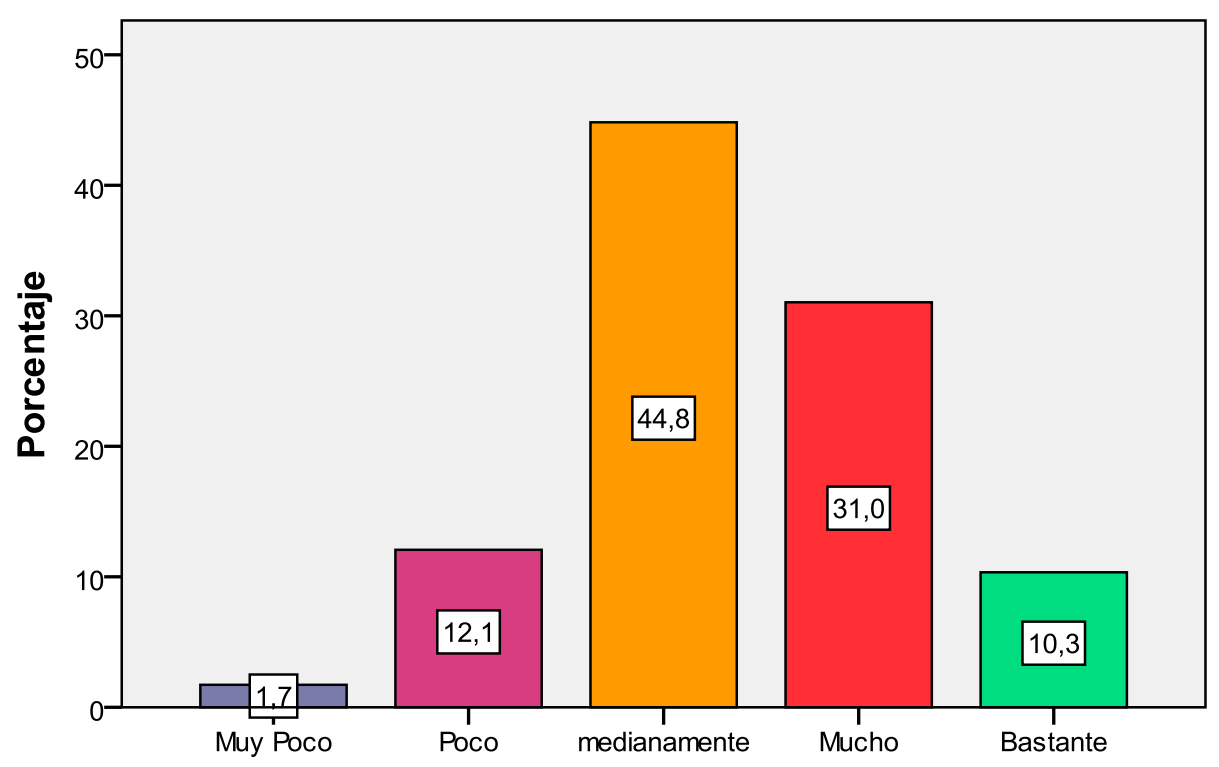




\section{¿Considera que puede acceder sin problemas al sistema virtual (plataforma) del curso?}

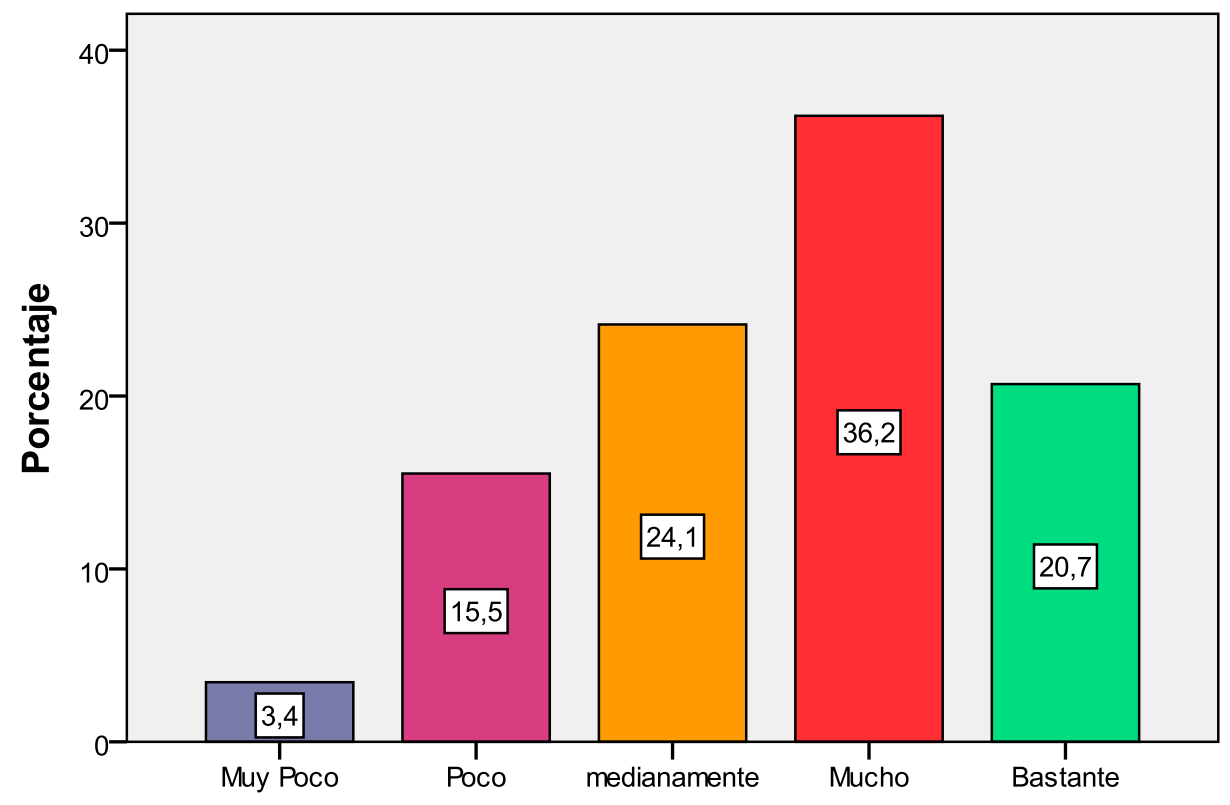

Fuente: Desarrollado con el software SPSS por autor.

En este caso aprecia que las puntuaciones se concentran en las categorías de (poco, medianamente y mucho), siendo creciente de poco a mucho, salvo la última pregunta donde se concentra la frecuencia en medianamente, lo que en este sistema el diseño de la plataforma no satisface a la mayoría de los usuarios.

\section{Análisis relacional de los sistemas de educación virtual y el Diseño de la Plataforma}

Con la finalidad de responder a la hipótesis propuesta y bajo la naturaleza de las variables, el método que se aplicará es la prueba de relación Chi-Cuadrada. El resultado de la prueba se expresa en razones de probabilidad y por último se verá el grado de relación. Esto nos permite predecir comportamientos futuros, que den sentido a las razones de probabilidad encontrada.

\section{Análisis Comparativo}

Como se toma los datos de la tesis de referencia para el análisis de la presente investigación, se detalla una tabla cruzada que involucra dos tipos de muestras tomadas en la tesis para hacer una comparación global de las satisfacciones percibidas por los estudiantes con respecto a las dimensiones Comunidad Colaborativa y Diseño de Plataforma para tener una visión del nivel de cada dimensión al observar el contraste entre un tipo de sistema y otro. 
Tabla N ${ }^{\circ}$ 7: Comparación de las dimensiones Comunidad Colaborativa y Diseño de la Plataforma de las muestras consideradas en la tesis de referencia

\begin{tabular}{lccc}
\hline \multirow{2}{*}{ Dimensión Colaboración } & \multicolumn{2}{c}{ Dimensión Diseño de la Plataforma } & \multirow{2}{*}{ Total } \\
\cline { 2 - 4 } & Insatisfecho & Satisfecho & 53 \\
\hline Insatisfecho & 38 & 15 & 75 \\
\hline Satisfecho & 11 & 64 & 128 \\
\hline Total & 49 & 79 & \multirow{2}{*}{} \\
\hline
\end{tabular}

Fuente: Desarrollado con el software SPSS por mi persona.

\section{Figura $N^{\circ}$ 5: Comparación de las dimensiones Comunidad Colaborativa y Diseño de Plataforma}

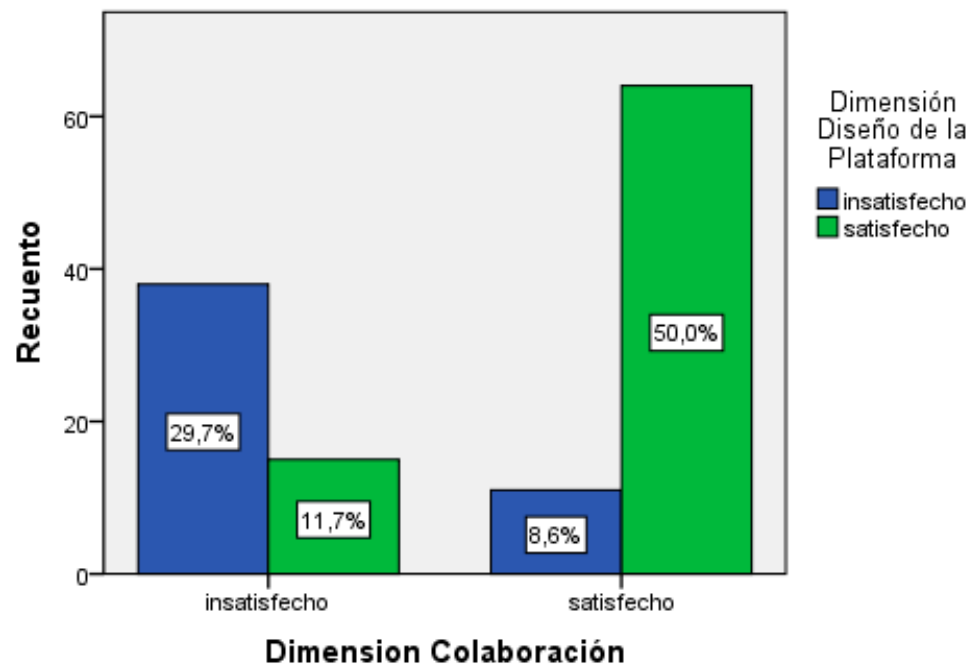

Fuente: Desarrollado con el software SPSS por autor.

En la tabla $\mathrm{N}^{\circ} 7$, se observa que existe una relación entre las dimensiones Comunidad Colaborativa y Diseño de Plataforma, encontrando que el $72 \%$ de los usuarios que están insatisfechos con la dimensión Comunidad Colaborativa también se encuentran insatisfechos con la dimensión Diseño de Plataforma. Por otro lado, el $85 \%$ de los que se encuentran satisfechos con la dimensión Comunidad Colaborativa también se encuentran satisfechos con la dimensión Diseño de Plataforma.

Esto muestra que la dimensión Diseño de la plataforma se relaciona con la dimensión Comunidad Colaborativa, lo que está supeditado a la interacción de los participantes y la flexibilidad de las plataformas o sistemas que sostienen todo el escenario virtual.

\section{Prueba de Relación}

Para probar de manera significativa que existe una relación entre las dimensiones Comunidad Colaborativa y Diseño de Plataforma, se plantea lo siguiente:

- Ho: No existe relación entre las dimensiones Comunidad Colaborativa y Diseño de Plataforma.

- Hr: Existe relación entre ambas variables. 
Tabla $N^{\circ}$ 8: Prueba Chi Cuadrada

\begin{tabular}{|c|c|c|c|c|c|}
\hline & Valor & gl & $\begin{array}{c}\text { Sig. asintótica } \\
\text { (bilateral) }\end{array}$ & $\begin{array}{c}\text { Sig. exacta } \\
\text { (bilateral) }\end{array}$ & $\begin{array}{l}\text { Sig. exacta } \\
\text { (unilateral) }\end{array}$ \\
\hline Chi-cuadrado de Pearson & $42,752^{a}$ & 1 &, 000 & & \\
\hline $\begin{array}{l}\text { Corrección por } \\
\text { continuidad }^{b}\end{array}$ & 40,372 & 1 & ,000 & & \\
\hline Razón de verosimilitudes & 44,663 & 1 &, 000 & & \\
\hline $\begin{array}{l}\text { Estadístico exacto de } \\
\text { Fisher }\end{array}$ & & & &, 000 &, 000 \\
\hline $\begin{array}{l}\text { Asociación lineal por } \\
\text { lineal }\end{array}$ & 42,418 & 1 &, 000 & & \\
\hline $\mathrm{N}$ de casos válidos & 128 & & & & \\
\hline
\end{tabular}

a. 0 casillas $(.0 \%)$ tienen una frecuencia esperada inferior a 5 . La frecuencia mínima esperada es 20.29 .

b. Calculado sólo para una tabla de $2 \times 2$.

Fuente: Desarrollado con el software SPSS por autor.

Para evaluar el nivel de significación de la prueba, compararemos si el p-value es menor a 0.05 (p-value < 0.05) lo que indica que la hipótesis nula será rechazada.

Para nuestro caso el nivel de significación de la prueba Chi-Cuadrada resulta menor que 0.05 lo que indica que rechazamos la hipótesis nula (Ho) "No existe relación entre las dimensiones Comunidad Colaborativa y Diseño de Plataforma", confirmando que existe relación entre ambas variables.
El método para saber si un escenario es mejor o más apropiado que el otro es la ChiCuadrada, el cual permite ver la relación que existe entre las dimensiones Comunidad Colaborativa y Diseño de Plataforma. Al ver mayor satisfacción con respecto a la dimensión Diseño de Plataforma cuando están satisfechos en la Dimensión Comunidad Colaborativa, se concluye que el nivel del diseño permite desarrollar más una comunidad colaborativa, lo cual favorece el aprendizaje. Esa es la relación que muestra la prueba finalmente.

Tabla $N^{\circ}$ 9: Tabla de clasificación

\begin{tabular}{|c|c|c|c|c|c|}
\hline & \multirow[b]{3}{*}{ Observado } & & \multicolumn{3}{|c|}{ Pronosticado } \\
\hline & & & \multicolumn{2}{|c|}{ Dimension Colaboración } & \multirow{2}{*}{$\begin{array}{l}\text { Porcentaje } \\
\text { correcto }\end{array}$} \\
\hline & & & insatisfecho & satisfecho & \\
\hline \multirow[t]{3}{*}{ Paso 1} & Dimension Colaboración & insatisfecho & 38 & 15 & 71,7 \\
\hline & & satisfecho & 11 & 64 & 85,3 \\
\hline & Porcentaje global & & & & 79,7 \\
\hline
\end{tabular}

a. El valor de corte es .500

Fuente: Desarrollado con el software SPSS por autor..

Previamente se mostró un panorama global del contraste de dos tipos de sistemas educativos virtuales estudiados en la tesis "Diseño de una Comunidad Colaborativa en los Sistemas Educativos Virtuales Tradicionales", para ver las diferencias de relaciones entre las dimensiones mencionadas. A continuación, se muestra el detalle de la relación de las dimensiones Comunidad Colaborativa y Diseño de Plataforma de la muestra elegida para este estudio porque refleja el perfil de estudiantes de derecho en un diplomado. 
Tabla N 10: Comparación de la dimensión Comunidad Colaborativa y Diseño de la Plataforma de la muestra seleccionada

\begin{tabular}{lccc}
\hline \multirow{2}{*}{ Dimensión Colaboración } & \multicolumn{2}{c}{ Dimensión Diseño de la Plataforma } & \multirow{2}{*}{ Total } \\
\cline { 2 - 3 } & Insatisfecho & Satisfecho & \\
\hline Insatisfecho & 37 & 11 & 48 \\
Satisfecho & 6 & 4 & 10 \\
\hline Total & 43 & 15 & 58 \\
\hline
\end{tabular}

Fuente: Desarrollado con el software SPSS por autor.

Figura $N^{\circ}$ 6: Comparación de las dimensiones Comunidad Colaborativa y Diseño de Plataforma de la muestra seleccionada.

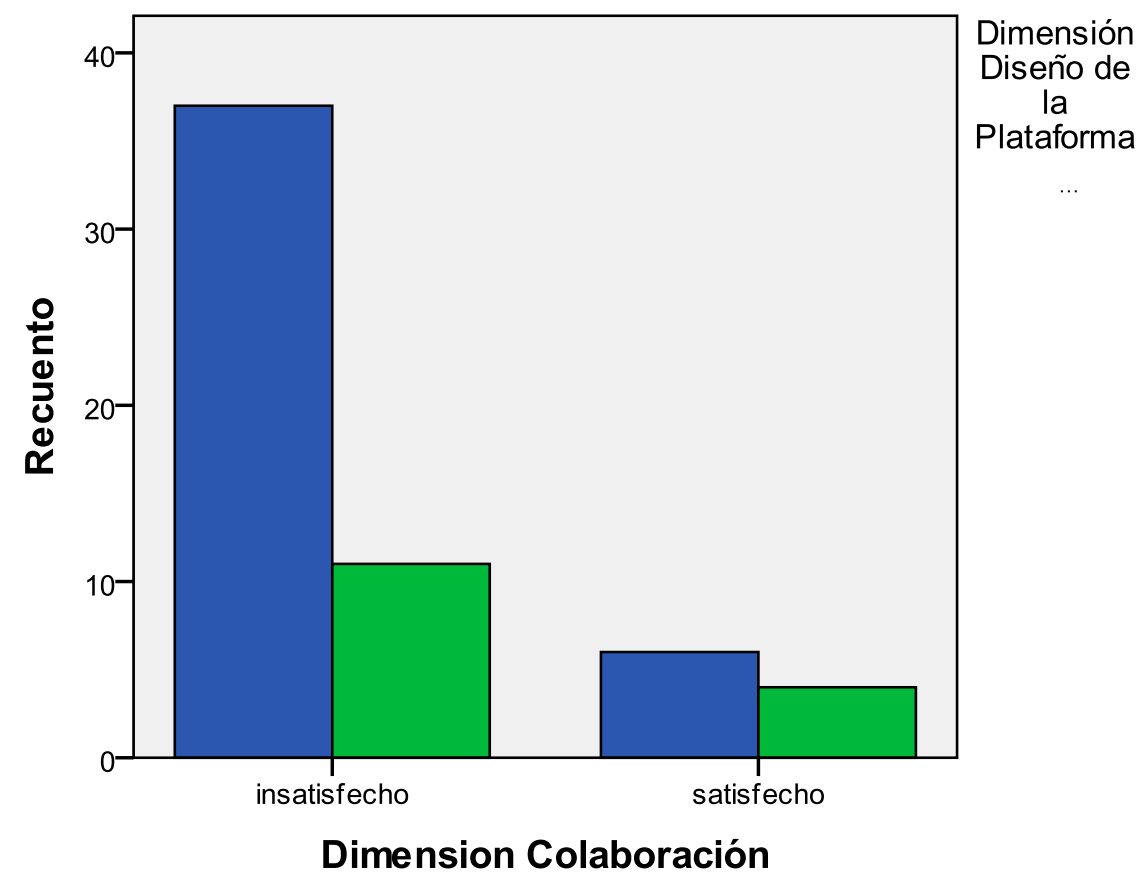

Fuente: Desarrollado con el software SPSS por autor.

En la tabla $\mathrm{N}^{\circ} 10$, se observa que existe una relación entre las dimensiones Comunidad Colaborativa y Diseño de Plataforma aplicado a la muestra seleccionada, encontrando que el $77 \%$ de los alumnos que están insatisfechos con la dimensión Comunidad Colaborativa, se encuentran insatisfechos con el Diseño de la Plataforma. Por otro lado, el $40 \%$ de los que se encuentran satisfechos con la dimensión Comunidad Colaborativa también se encuentran satisfechos con la dimensión Diseño de Plataforma.

Esto muestra que el Diseño de Plataforma se relaciona con la Dimensión Comunidad Colaborativa de una manera poco positiva en el sistema de educación virtual correspondiente a la muestra seleccionada porque para la mayoría el diseño de la plataforma propicia una comunidad de colaboración. 


\section{DISCUSIÒN}

La presente investigación como se mencionó anteriormente pretende mejorar lo realizado por el investigador en el trabajo "Diseño de una Comunidad Colaborativa en los Sistemas Educativos Virtuales Tradicionales" en el 2016. En aquel año se tomó dos muestras pertenecientes a dos poblaciones respectivamente que hacen referencia a dos tipos de sistemas educativos virtuales: tradicionales y contemporáneos. Se intentó medir el nivel de colaboración en las muestras de los dos tipos de sistemas mediante la percepción de los mismos participantes para corroborar si entornos más flexibles y con una comunidad más solidaria propician el desarrollo de un aprendizaje colaborativo en un entorno virtual. Se había definido la dimensión comunidad colaborativa para poder analizarla en las dos muestras

Efectivamente en los resultados se observó que entornos con una comunidad más solidaria obtuvieron mayor aceptación por parte de los participantes quienes expresaron una satisfacción mayor con respecto al entorno virtual que soportaba su programa académico, sin embargo, en la presente investigación se evaluó la posibilidad de determinar que elemento o dimensión podría tener relación o influencia en la dimensión comunidad colaborativa. De acuerdo a las investigaciones de referencia se decidió probar si el nivel del diseño de una plataforma tenía efecto o relación con el nivel de colaboración de una comunidad virtual. El nivel de colaboración fue definido en el trabajo "Diseño de una Comunidad Colaborativa en los Sistemas Educativos Virtuales Tradicionales" por el investigador.

La búsqueda de calidad es un acto constante en la educación y no puede ser dejado de lado en el campo virtual, por ende, los estándares evolucionan para cuidar al estudiante como el elemento que debe ser más beneficiado en todo el proceso de aprendizaje.

De tal forma, para la presente investigación es importante rescatar el nivel de calidad del diseño de la plataforma como un aspecto sustancial para poder facilitar el acceso flexible, la generación de actividades, e intercambio de conocimiento. Por tal motivo se define la dimensión diseño de plataforma como un valor agregado a lo trabajado en la investigación "Diseño de una Comunidad Colaborativa en los Sistemas Educativos Virtuales Tradicionales", la cual para la presente investigación es importante potenciar para encontrar una explicación más concreta con respecto a una posible relación con otro factor.

Se establecieron los objetivos específicos de determinar la dimensión Diseño de la Plataforma en los sistemas educativos virtuales y determinar la relación entre el nivel de calidad de Diseño de la Plataforma y el nivel de Comunidad Colaborativa en un entorno educativo virtual. Estos objetivos establecen el camino en la investigación para hacer la prueba de hipótesis y poder comprobar la posible relación entre las dimensiones comunidad colaborativa y diseño de plataforma. Para la dimensión comunidad colaborativa se midió su nivel mediante la percepción de los estudiantes mismos y quedaba para la presente investigación reformular la dimensión diseño de plataforma para medir su nivel de calidad.

En la investigación "Diseño de una Comunidad Colaborativa en los Sistemas Educativos Virtuales Tradicionales" realizada en el 2016 se tenía una dimensión diseño de plataforma propuesta por el autor, pero no fue trabajada ni considerada para poder concluir un aporte con relación a la importancia del aprendizaje colaborativo en entornos virtuales. Para la presente investigación se considera importante enfocar la relación entre ambas dimensiones para probar la hipótesis establecida y por ende se restructura la dimensión diseño de plataforma para fortalecer los resultados y poder generar un mejor aporte a la comunidad.

Para darle una determinación a la dimensión propuesta se aplicó el análisis factorial con la finalidad de probar que las 5 variables seleccionadas formen un mismo constructo que nombró "Diseño de la Plataforma". Este constructo dio la solidez a la dimensión sugerida para proseguir con las validaciones. El resultado del análisis factorial, que brinda la consistencia de la dimensión propuesta, muestra la matriz de varianza explicada, que corrobora que estas 5 variables se están asociando a un solo constructo y que este explica el $73 \%$ de la variabilidad de esta variable subyacente "Diseño de la Plataforma". La matriz de componentes muestra los coeficientes (aporte de las variables al constructo), es decir expresa 
como la variable 1 y la 3 presentan mayores índices debido a que estas variables aportan más en la variabilidad de la dimensión Diseño de la Plataforma.

Posteriormente cuando se vio la sostenibilidad del nuevo constructo como dimensión fuerte a medir (diseño de plataforma), se realizó un análisis descriptivo de los ítems que componen la dimensiones comunidad colaborativa y diseño de la plataforma, para ambos sistemas de educación virtual.

En la dimensión Comunidad Colaborativa del escenario virtual considerado se observó que la puntuación media en cada pregunta de dimensión no dista mucho de su mediana con lo que la percepción es casi homogénea, salvo las dos primeras preguntas (establecen foros y comunicación fluida), cuyos valores mínimos y máximos son extremos. Se aprecia que las puntuaciones se concentran en las categorías de (poco, medianamente y mucho), siendo la de mayor frecuencia "medianamente", lo que implica que este sistema tiene una naturaleza más rígida y no se tiene muy desarrollado la dimensión de Comunidad Colaborativa.

En la dimensión Diseño de Plataforma del escenario virtual mencionado se observó que la puntuación media y mediana en cada pregunta no dista mucho, con lo que la percepción es casi homogénea, sin embargo, la pregunta dos y tres (Descarga y Acceso), presentan menor diferencia entre sus medias y medianas. Se aprecia que las puntuaciones se concentran en las categorías de (poco, medianamente y mucho), siendo creciente de poco a mucho, salvo la última pregunta donde se concentra la frecuencia en "medianamente", lo que conduce a desprender que el diseño de la plataforma no satisface a la mayoría de los usuarios.

Posteriormente, con la finalidad de responder a la hipótesis propuesta y bajo la naturaleza de las variables, el método que se aplicó es la prueba de relación chi-cuadrado. El resultado de la prueba se expresó en razones de probabilidad y por último se vio el grado de relación. Esto permitió predecir comportamientos futuros, para dar sentido a las razones de probabilidad encontradas.

Para la prueba se elaboró una tabla cruzada (cuadro comparativo) para mostrar las relaciones entre sistema de educación virtual y la dimensión diseño de la plataforma. En una tabla de la investigación se observó que existe una relación entre las dimensiones comunidad colaborativa y diseño de la plataforma, encontrando que el $72 \%$ de los usuarios que están insatisfechos con la dimensión colaboración también se encuentran insatisfechos con el diseño de la plataforma.

En una tabla expuesta en la presente investigación se observó que existe una relación entre las dimensiones Comunidad Colaborativa y Diseño de la Plataforma del escenario virtual tomado como estudio, encontrando que el $77 \%$ de los estudiantes que están insatisfechos con la dimensión colaboración, se encuentran insatisfechos con el diseño de la plataforma. Por otro lado, el $40 \%$ de los que se encuentran satisfechos con la dimensión comunidad colaborativa también se encuentran satisfechos con la dimensión diseño de la plataforma. Esto muestra que el Diseño de la Plataforma se relaciona con la dimensión colaborativa de una manera poco positiva en el sistema de educación virtual tomado porque para la mayoría el DISEÑO de la Plataforma no genera una comunidad de colaboración.

Las pruebas realizadas permitieron establecer una validez a la hipótesis mencionada $\mathrm{y}$ poder verificar que efectivamente existe una relación entre el nivel de calidad de Diseño de una Plataforma y el nivel de la dimensión de Comunidad Colaborativa. Se hace énfasis en la palabra "nivel" para poder establecer las mediciones respectivas de las dimensiones y posteriormente evaluar los resultados.

Las acciones deben ser llevadas para generar un ambiente colaborativo dentro de un entorno virtual, pero deben estar basadas en metodologías y estrategias para generar una motivación. La motivación es muy importante para el participante y algo que siempre mantiene activo al mismo es la competitividad. Es por ello que al margen de tener un diseño que motive al participante, es sumamente vital que el moderador o docente pueda propiciar un aprendizaje más colaborativo. Es por ello que el diseño de una plataforma también es importante para soportar las nuevas propuestas y dinámicas.

La presente investigación rescató el nivel de diseño de una plataforma como relevante 
para soportar un aprendizaje colaborativo, si bien es cierto en la investigación citada al inicio se evaluó la percepción de Comunidad Colaborativa, se planteó ir más allá en su aproximación a explicar las razones de una apreciación más colaborativa en los escenarios virtuales. Por tal motivo se determinó una nueva dimensión llamada Diseño de Plataforma, para evaluarla desde su nivel de calidad mediante los mismos estudiantes y así establecer una probable relación que pudo corroborarse al final.

Mientras más actividades permita generar el diseño de una plataforma es más probable que el alumno este motivado y pueda establecer interacciones con sus demás compañeros y con el docente. Si bien es cierto no se da en todas las instituciones educativas el hecho de que se potencie el nivel de calidad del diseño de una plataforma para soportar la educación virtual, sería conveniente cada cierto tiempo evaluar las políticas para una reestructuración del Diseño de Plataforma teniendo en cuenta de que el análisis de mejora debe ser continuo para el beneficio del estudiante.

\section{CONCLUSIONES}

Entre las conclusiones que se puede desprender en el presente trabajo de investigación tenemos:

- Un diseño flexible de plataforma virtual enfocado en el estudiante favorece a que pueda desarrollarse un aprendizaje colaborativo en la enseñanza del derecho debido a que posibilita una mejor interacción entre los participantes. Fomentar el intercambio de ideas mediante las actividades o dinámicas es vital para consolidar conocimientos porque desde tiempo atrás la construcción de soluciones y modelos nuevos se basó en los propios seres humanos. Es por ello que para soportar una definición de ideas y conceptos se necesita tener el escenario lo más idóneo posible.

- Un escenario óptimo implica una accesibilidad y la posibilidad de usar los recursos de la plataforma lo más fácil posible para poder interactuar. La claridad en el acceso y una interface amigable guía mejor al estudiante para poder corresponder a las actividades propuestas por el docente sin mucho rechazo. El docente a su vez aprovecha la flexibilidad de una plataforma para generar actividades y motivar a su comunidad como moderador.

- Existe una relación entre la dimensión comunidad colaborativa y dimensión diseño de plataforma definida en la investigación. En la muestra del sistema educativo virtual elegido para el estudio se percibió una insatisfacción significativa con el diseño de la plataforma y de igual manera por la dimensión comunidad colaborativa. Un diseño poco flexible distancia al estudiante en su rol de ser un elemento participativo. Siguiendo con la investigación realizada, no solo se observó un rechazo en la dimensión comunidad colaborativa, sino que la dimensión propuesta de diseño de plataforma también recibió poca consideración en los puntajes, de tal forma que guardan cierta relación probada con la estadística respectiva.

- Ambas dimensiones, tanto diseño de plataforma como comunidad colaborativa, se relacionan y guardan congruencia porque si partimos de los antecedentes definidos en esta investigación que sostienen que un buen diseño de plataforma posibilita mejorar un aprendizaje colaborativo en una comunidad virtual, entonces mediante la prueba estadística chi-cuadrado se puede decir que se confirmó la relación entre ambas dimensiones.

- Necesariamente el trabajo colaborativo favorece al aprendizaje y más aún si es un entorno virtual, pero tiene que tener las mejores condiciones para permitir una comunicación aceptable. Las políticas institucionales van a determinar el camino en las mejoras para la modalidad virtual en cualquier rama educativa y siempre se espera que las propuestas tecnológicas potencien dichas pautas por el beneficio del estudiante.

\section{RECOMENDACIONES}

- Considerar al estudiante como el elemento principal al momento de establecer un proyecto virtual para soportar un programa educativo. El estudiante debe ser el más beneficiado en el escenario virtual y las instituciones educativas deben procurar supervisar que se estén estableciendo todos los recursos que permitan que el alumno 
prospere en el campo del conocimiento y así evitar la desestimación futura de las propuestas tecnológicas en el campo de la educación.

- Tener en cuenta modelos de dinámicas para apoyar aún más la construcción de conocimiento en la enseñanza del derecho. Es importante mantener la motivación entre los participantes a través de un ambiente más amigable para favorecer la discusión de temas de interés. La motivación resulta vital y parte desde la propuesta del docente y se sostiene con la tecnología que abre el camino de desarrollar nuevos caminos.

- Se recomienda considerar lo expuesto en el trabajo dela investigación realizado por mi persona en el 2016, en la cual se propone considerar los factores de competencia, puntos y reconocimientos de la teoría de gamificación para implementar actividades que promuevan el trabajo en equipo bajo un entorno que conecte más al estudiante. El reconocimiento siempre motiva al estudiante y no debe perderse eso de vista si queremos que los participantes se mantengan conectados a un programa virtual. Normalmente estamos acostumbrados a una manera tradicional de llevar los escenarios virtuales, quizás como repositorio de información, pero debería evolucionar esa manera de plantear las propuestas virtuales $\mathrm{y}$ apostar por un rediseño tecnológico que soporte un escenario más interactivo donde pueda mantenerse la competitividad.

- Se recomienda explorar otros aspectos relacionados a la educación virtual y sus repercusiones otras ramas educativas para poder dar un contraste significativo y esclarecer más los niveles de adaptación de cada campo educativo. El docente resulta otro aspecto que podría estudiarse desde su perspectiva para poder proponer mejoras y soluciones que permita que ellos también se sientan involucrados con las nuevas tendencias tecnológicas.

\section{FUENTES DE INFORMACIÓN}

A. Daelle \& C. Brassard. (2003). Communautés d'apprentissage dans l'enseignement supérieur: objectifs et conditions de développement. Congreso Les communautés virtuelles d'apprentissage. 2e colloque de Guéret.
Aretio, L. G. (1999). Fundamento y Componentes de la Educación a Distancia. Revista JIberoaméricana de Educación a Distancia.

Aretio, Lorenzo García. (1989). La educación. Teorías y conceptos - Madrid.

Barab, S. A. (2003). An Introduction to the Special Issue: Designing for Virtual Communities in the Service of Learning. The Information Society, 197-201.

Cabero, J. (2006). Comunidades virtuales para el aprendizaje. Su utilización en la enseñanza. Edutec. Revista Electrónica de Tecnología Educativa Núm. 20/ Enero 06. Obtenido de http://www.uib.es/depart/gte/gte/edutec-e/ revelec20/cabero20.htm

Carbonell. (2011). Abogados y Estudiantes de Derecho frente a las TIC. Recuperado de https://educacionyculturaaz.com/abogados-yestudiantes-de-derecho-frente-a-las-tic/

Cesteros, Ana y Fernández, Pampillón. (2009). Las plataformas e-learning para la enseñanza y el aprendizaje universitario en Internet. Madrid, España: Universidad Complutense de Madrid.

Coll, C. (2004). Las comunidades de aprendizaje. Nuevos horizontes para la investigación y la intervención en psicología de la educación. Obtenido de http:// www. ub.es/grintie/GRINTIE/Library/public/CC_ Almeria_04.pdf

Daele, A. (2004). Développement professionnel des enseignants dans un contexte de participation à une communauté virtuelle: une étude exploratoire.

Fabela. (2015). Técnicas de Muestreo y Prueba de Hipótesis. Recuperado de https:// es.slideshare.net/ErnestoFabela1196/u2tecnicas-de-muestreo-y-pruebas-de-hipotesis

F. Henri \& J. Basque. (2003). Conception d'activités d'apprentissage collaboratif en monde virtuel, en Collaborer pour apprendre et faire apprendre. DEAUDELIN C. \& NAULT, T. (Eds.). SaintFoy: PUQ.

Florido Bacallao, R.y Florido Bacallao, M. (2003). La Educación a distancia, sus retos y posibilidades. Granada, España: Centro de Referencia para la Educación Avanzada (CREA)- Cuba. 
García, G. R. (2006). Antecedentes históricos de la Educación a Distancia. COORDINACIÓN DE UNIVERSIDAD ABIERTA Y EDUCACIÓN A DISTANCIA.

Galindo González, Rosa María; Martínez de la Cruz, Nadia Livier \& Ruíz Aguirre, Edith Inés. (2015). El aprendizaje colaborativo en ambientes virtuales

García, G. R. (2006). Antecedentes históricos de la Educación a Distancia. COORDINACIÓN DE UNIVERSIDAD ABIERTA Y EDUCACIÓN A DISTANCIA.

Gonzales Rus, Juan José. (2003). Reflexiones sobre el futuro de la enseñanza del derecho y sobre la enseñanza del derecho en el futuro.

Guérin, F. (2004). Le concept de communauté: une illustration exemplaire de la production des concepts en sciences sociales? Paper presented at the $13^{\mathrm{a}}$ Conférence de l'AMI, Normandia . Vallé de Seine.

Lorenzo García Aretio \& R. Medina Rubio. (1993). Guía didáctica de Teoría de la educación. Madrid: UNED p.56.

M. Pazos, Pérez \& J. Salinas. (2001). Comunidades Virtuales: De las listas de discusión a las comunidades de aprendizaje. Obtenido de http://gte.uib.Es/articulos/ cvirtuales01.pdf

Manuel Meirinhos \& António Osório. (2009). Las Comunidades Virtuales de Aprendizaje: El Papel Central de Colaboración. Pixel-Bit. Revista de Medios y Educación, 45-60.

McConnel, D. (2006). E-learning Groups and Communities. New York. McGraw-Hill.

Moreno Salamanca, Mariac (2013). La flexibilidad didáctica en entornos virtuales de aprendizaje.
Ossiannilsson, E. (2012). Benchmarking E-Learning in Higher Education - Lessons learned from international projects. Acta Universitatis Ouluensis - C Tec h n i c a 436.

P. Dillenbourg, C. Poirier, \& L. Carles, . (2003). Communautés virtuelles d'apprentissage: e-jargon ou nouveau paradigme? L'essor des communautés virtuelles d'apprentissage.

Ramboll, P. (2004). Studies in the Context of the E-learning Initiative: Virtual Models of European Universities (Lot ). Draft Final Report to the EU Commission,DG Education y Culture". Obtenido de http:// www.elearningeuropa.info/extras/pdf/virtual_ models.pdf.

Rena M. Palloff \& Keith Pratt. (2005). Collaborating Online: Learning Together in Community.

Rheingold, H. (1996). La comunidad virtual. Una sociedad sin fronteras.

Romiszowski, A. J. (2004). "How's the E-learning Baby? Factors Leading to Success or Failure of an Educational Technology". Innovation Educational Technology 44(1), 5-27 consultado agosto 2008. Obtenido de http:// www.elearning-reviews.org/topics/resourcesmanagement/project-management/2004romiszo

Salinas Ibáñez, J. (2004). Entornos virtuales y formación flexible. 